\title{
PORTAL VEIN TUMOR THROMBUS OF LIVER METASTASIS FROM LUNG CANCER
}

\author{
Ryoko Ogawa ${ }^{1,2}$, Takahide Kodama ${ }^{1}$, Koichi Kurishima ${ }^{2}$, Katsunori Kagohashi' ${ }^{2}$ Hiroaki Satoh ${ }^{2}$ \\ Division of Respiratory Medicine, Ryugasaki Saiseikai Hospital, Japan ${ }^{1}$; Divisions of Respiratory Medicine, Institute \\ of Clinical Medicine and Mito Medical Center, University of Tsukuba, Japan ${ }^{2}$
}

\begin{abstract}
Summary: We report a case of liver metastasis of lung carcinoma with portal vein tumor thrombus (PVTT). Although the primary lesion of lung tumor remained unchanged, the patient rapidly developed wide-spread metastases and formed PVTT of liver metastasis. The primary lesion showed features of mixed Clara and bronchial surface epithelial cell component type adenocarcinoma with small foci of micropapillary pattern. Micropapillary pattern was observed in the metastatic lesions in the liver and PVTT. Micropapillary pattern lung adenocarcinoma may develop rapid metastases and cause PVTT associated with liver metastasis. We should perform a detailed examination to establish correct diagnosis.
\end{abstract}

Key words: Portal vein tumor thrombus; Lung adenocarcinoma; Micropapillary pattern

\section{Introduction}

Portal vein tumor thrombus (PVTT) is a rare complication of metastatic liver tumors $(4,8,15)$. We show herein the first case report describing PVTT associated with hepatic metastasis of lung adenocarcinoma. In addition to this, our case showed micropapillary pattern, which has recently been recognized as a histologic subtype with poor prognostic significance $(2,7,10,12)$, in the metastatic lesions.

\section{Case report}

A 67-year old man with advanced lung adenocarcinoma admitted to our hospital complaining general fatigue. Nine months before the admission, he was diagnosed as having metastatic lung adenocarcinoma. The patient received 4 courses of chemotherapy with carboplatin and paclitaxel, but achieved no response. Then, he had gefitinib for 2 months, but the response was evaluated as stable disease. Thereafter, he did not continue to receive any chemotherapy and was not followed up regularly. On admission, chest radiograph and $\mathrm{CT}$ scan revealed a mass lesion, $20 \mathrm{~mm}$ in diameter, in the left upper lobe of the lung with mediastinal lymph node swelling.

Physical examinations revealed unremarkable and hepatitis B surface antigen and antibody to hepatitis $C$ virus were negative, but the levels of aspartate aminotransferase (329 IU/1), alanine aminotransferase (220 IU/1), and total bilirubin $(1.8 \mathrm{mg} / \mathrm{dl})$ were elevated. Elevated serum levels of carcinoembryonic antigen $(2480 \mathrm{ng} / \mathrm{ml})$ and CYFRA21-1 $(8.6 \mathrm{ng} / \mathrm{ml})$ were observed. Abdominal ultrasonographic examination showed a large mass in the right lobe of the liver and occlusion of the intrahepatic portal veins. Abdominal CT scan showed large well-defined parenchymal perfusion abnormalities in whole left lobe and in some segments of right lobe of the liver, respectively (Fig. 1). Abdominal CT also showed several tumors measuring 10-20 mm in the right lobe of the liver. Based on the patient's history, negative hepatitis virus markers, and radiologic findings, we considered that liver metastasis of lung adenocarcinoma was as the most likely diagnosis.

Subsequently, the patient's condition rapidly deteriorated with progressive jaundice, and he died of hepatic failure 2 months after the admission. An autopsy was performed. The mass in the left upper lobe of the lung, measuring $20 \mathrm{~mm}$ in diameter, was confirmed histologically as mixed Clara and bronchial surface epithelial cell component type of adenocarcinoma with small foci of micropapillary pattern. Macroscopic examination of the liver showed a large well-defined necrotic area in the both lobes, which was confirmed as obstruction of portal venous blood flow (Fig. 2). Pathological findings of cancer cells in the portal tumor thrombus as well as liver tumors shared same features with that of micropapillary morphology of lung adenocarcinoma, and the diagnosis of liver metastasis from lung adenocarcinoma was confirmed (Fig. 3). Same micropapillary pattern adenocarcinoma was observed in the metastatic lesions in the peritoneum, pleura, lung, and bone marrow. 


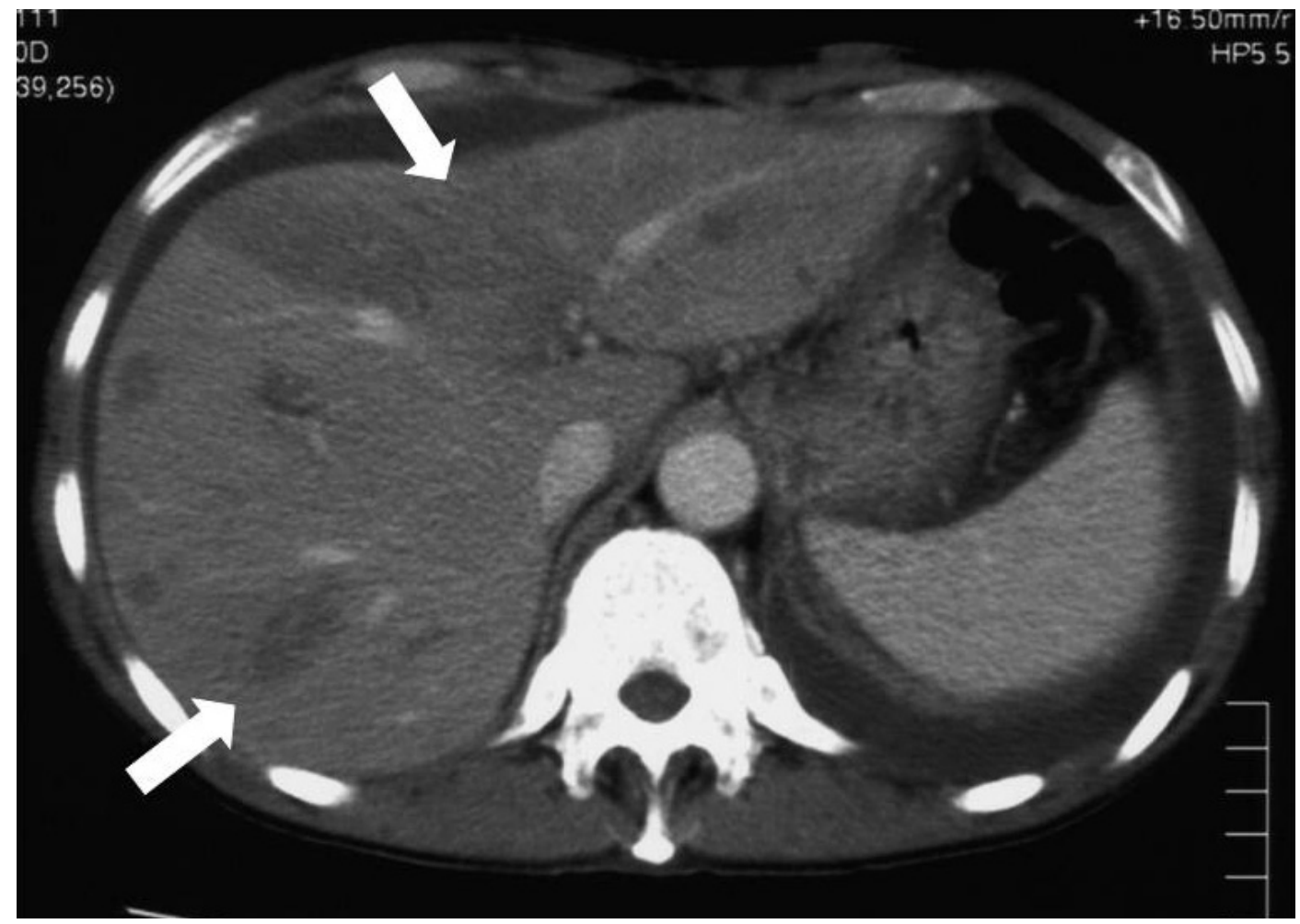

Fig. 1: Abdominal CT scan showed a large well-defined homogenous low density area in the left and small these areas in the right lobe of the liver, which was later confirmed as obstruction of portal venous blood flow.

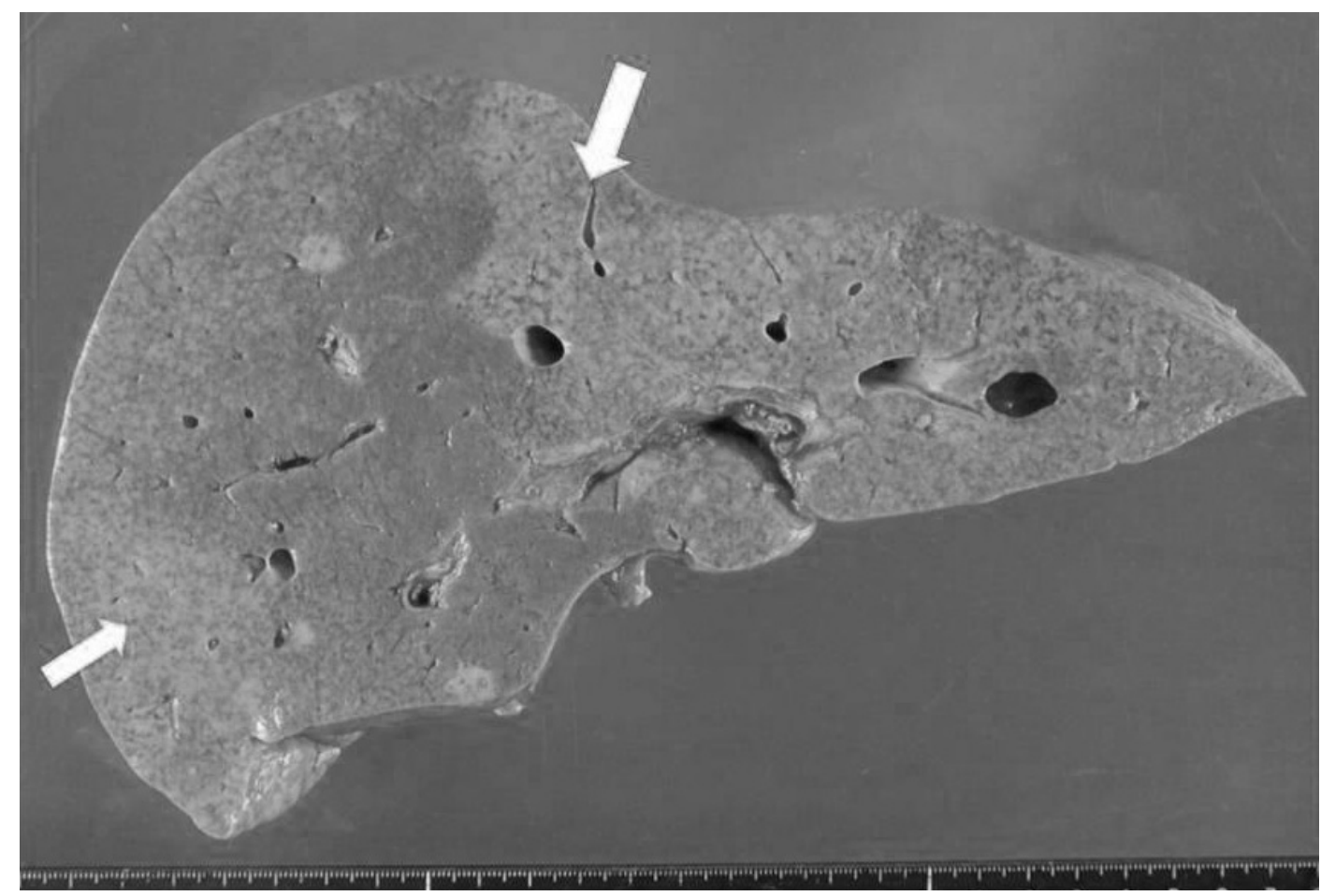

Fig. 2: Macroscopic examination at autopsy showed a large well-defined necrotic area in the both lobes of the liver, which was microscopically confirmed as obstruction of portal venous blood flow. 


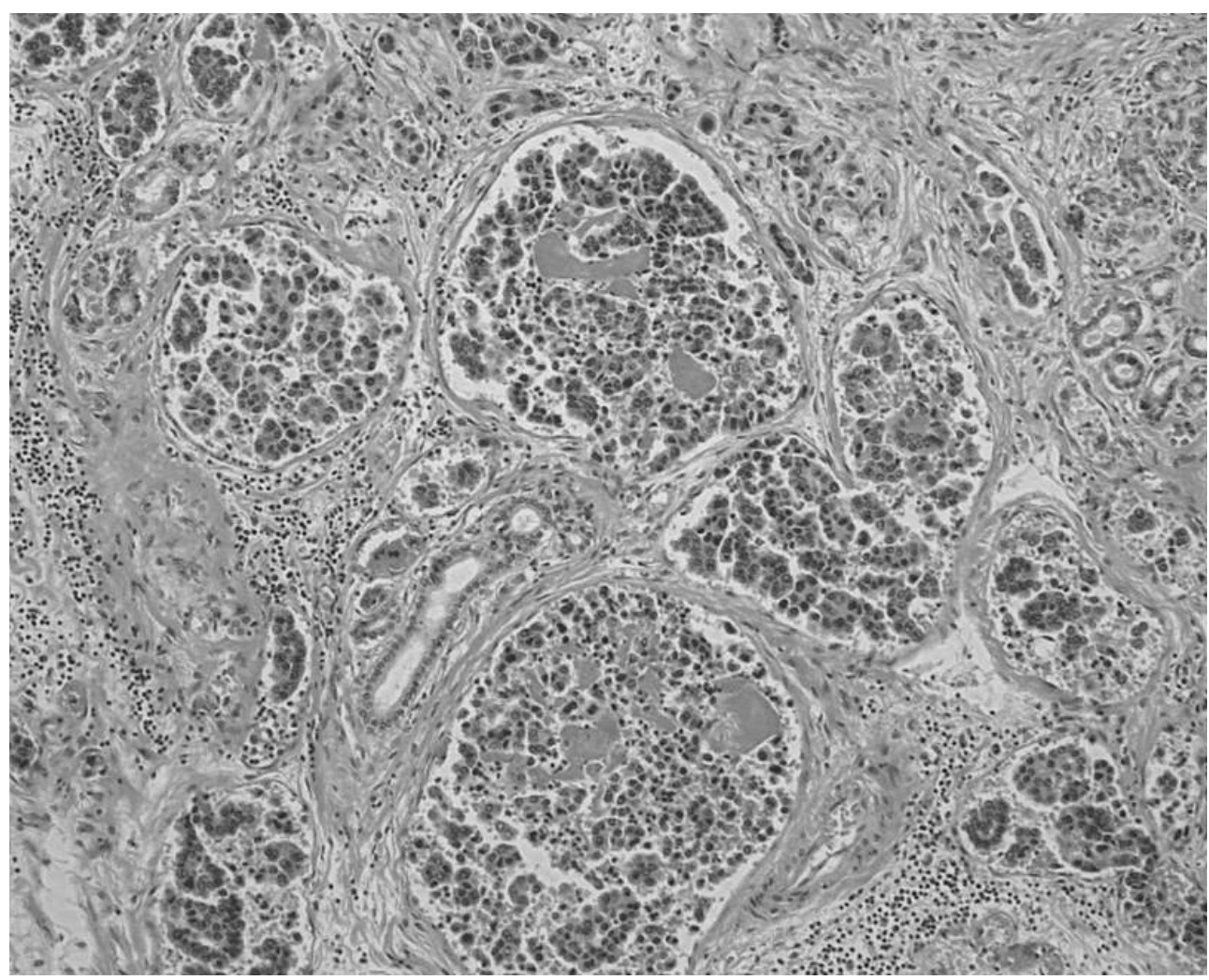

Fig. 3: Tumor cells in the portal tumor thrombus shared histologic features with that of lung adenocarcinoma with micropapillary pattern, and the diagnosis of liver metastasis from lung adenocarcinoma was confirmed (x400, Hematoxylin-Eosin Stain).

\section{Discussion}

PVTT is a rare complication of metastatic liver tumor in clinical practice $(4,8,15)$. Although Atri et al reported that PVTT was found in $8 \%$ of patients with liver metastasis (3), the precise incidence of PVTT complicating liver metastasis remains unknown. Cancers in gastrointestinal tract are the most common primary sources of hepatic metastases causing PVTT, however, cases of other malignancies such as melanoma, kidney cancer, pancreas cancer also have been reported $(3,4,8,13,15)$. To our knowledge, this is the first case report describing PVTT associated with hepatic metastasis of lung adenocarcinoma. In addition to this, it is notable that small foci of micropapillary pattern lung adenocarcinoma cells caused PVTT and metastasized to various organs.

The presence of PVTT indicates poor prognosis $(4,8$, 15). It often leads to extensive spreading of the tumor throughout the liver, and can increase portal venous blood pressure, resulting in the fatal rupture of esophageal varices, and can decrease portal flow which causes ascites, jaundice, hepatic encephalopathy, and liver failure $(4,8$, 15). Diagnosis of tumor thrombus is mainly made by radiologic examinations because it is mostly asymptomatic and clinically unsuspected. It is well known that CT is useful for the depiction of the anatomy and pathology of the portal vein. The liver has dual blood supply from the hepatic artery and portal vein, and receives $70 \%$ to $80 \%$ of its blood flow from the latter. When portal venous blood flow is decreased in the liver parenchyma, arterial blood supply may become dominant. The wedge-shaped perfusion abnormalities on CT are probably due to the stoppage of portal venous flow by PVTT $(4,5,8)$, as shown in our case.

The micropapillary morphology of lung adenocarcinoma has been recognized increasingly as a histologic subtype with poor prognostic significance $(2,7,10,12)$. The micropapillary variant of carcinoma not only in lung but also in other organs appear to be associated with invariably with a higher degree of aggressiveness, manifested by high stage at presentation, massive lymph node metastasis, and extensive lymphovascular invasion $(1,2,6,10,11)$. Very recently, Sánchez-Mora evaluated the prognostic significance of the micropapillary pattern of lung adenocarcinoma. They reported the micropapillary component proved to be an independent prognostic factor in multivariate survival analysis (14). Therefore, it is likely that micropapillary carcinoma shows an aggressive behavior because of the intensive nature of the invasive growth. In our patient, the autopsy revealed a mass lesion, $20 \mathrm{~mm}$ in largest diameter, in the upper lobe of the left lung. Microscopically, the pri- 
mary lesion mostly showed features of mixed Clara and bronchial surface epithelial cell component type adenocarcinoma with small foci of micropapillary pattern. Same micropapillary pattern was observed in the metastatic lesions in the liver, peritoneum, pleura, lung, bone marrow, and portal tumor thrombus. Our patient also showed an aggressive clinical course after the chemotherapy with gefitinib. It has been recently reported the possibility of fatal rapid recurrence after a disease control by this drug (9). We could not know why our lung adenocarcinoma patient developed PVTT, which has been very rare complication of metastatic liver tumors. In addition, we could not explain why the patient deteriorated rapidly and small foci of micropapillary pattern lung adenocarcinoma cells had rapid wide-spread metastases, although the primary lesion remained unchanged. Future biological and clinicopathological studies will provide correct answers.

In summary, we presented an unusual case of lung adenocarcinoma with small foci of micropapillary pattern that had rapid wide-spread metastases and formation of extensive tumor thrombus in the portal venous system. Although very rare, we should be alert to rapid deterioration of patients with PVTT and should perform a detailed examination to establish correct diagnosis.

\section{References}

1. Amin MB, Ro JY, el-Sharkawy T, et al. Micropapillary variant of transitional cell carcinoma of the urinary bladder. Histologic pattern resembling ovarian papillary serous carcinoma, Am J Surg Pathol 1994; 18: 1224-32.
2. Amin MB, Tamboli P, Merchant SH, et al. Micropapillary component in lung adenocarcinoma: a distinctive histologic feature with possible prognostic significance, Am J Surg Pathol 2002; 26: 358-64.

3. Atri M, de Stempel J, Bret PM, Illescas FF. Incidence of portal vein thrombosis complicating liver metastasis as detected by duplex ultrasound. J Ultrasound Med 1990; 9: 285-9.

4. Ito K, Higuchi M, Kada T, et al. CT of acquired abnormalities of the portal venous system. Radiographics 1997; 17: 897-917.

5. Ito K, Honjo K, Fujita T, Awaya H, Matsumoto T, Matsunaga N. Hepatic parenchymal hyperperfusion abnormalities detected with multisection dynamic MR imaging: appearance and interpretation. J Magn Res Imaging 1996; 6: $861-7$.

6. Luna-More S, Gonzalez B, Acedo C, Rodrigo I, Luna C. Invasive micropapillary carcinoma of the breast. A new special type of invasive mammary carcinoma. Pathol Res Pract 1994; 190: 668-74.

7. Makimoto $\mathrm{Y}$, Nabeshima K, Iwasaki $\mathrm{H}$, et al. Micropapillary pattern: a distinct pathological marker to subclassify tumours with a significantly poor prognosis within small peripheral lung adenocarcinoma $(\leq 20 \mathrm{~mm})$ with mixed bronchioloalveolar and invasive subtypes (Noguchi's type C tumours). Histopathology 2005; 46: 677-84.

8. Marn C, Francis I. CT of portal venous occlusion. AJR 1992; 159: 717-26.

9. Miyazaki K, Ano T, Nakazawa K, Satoh H, Ohtsuka M. Late fatal recurrence in gefitinib-treated NSCLC patients. Tuberk Toraks 2007; 55: 400-3.

10. Miyoshi T, Satoh Y, Okumura S, et al. Early-stage lung adenocarcinomas with a micropapillary pattern, a distinct pathologic marker for a significantly poor prognosis. Am J Surg Pathol 2003; 27: 101-9.

11. Nagao T, Gaffey TA, Visscher DW, et al. Invasive micropapillary salivary duct carcinoma: a distinct histologic variant with biologic significance, Am J Surg Pathol 2004; 28: 319-26.

12. Roh MS, Lee JI, Choi PJ, Hong YS. Relationship between micropapillary component and micrometastasis in the regional lymph nodes of patients with stage I lung adenocarcinoma, Histopathology 2004; 45: 580-6.

13. Saiura A, Umekita N, Matsui Y, et al. Successful surgical resection of solid and cystic tumor of the pancreas with multiple liver metastases and a tumor thrombus in the portal vein. Hepatogastroenterology 2000; 47: 887-9.

14. Sánchez-Mora N, Presmanes MC, Monroy V, et al. Micropapillary lung adenocarcinoma: a distinctive histologic subtype with prognostic significance. Case series. Hum Pathol. 2008; 39: 324-30.

15. Tanaka A, Takeda R, Mukaihara S, et al. Case report: Tumor thrombi in the portal vein system originating from gastrointestinal tract cancer. J Gastroenterol 2002; 37: 220-8.

Received: 25/09/2009.

Accepted: 10/12/2009.

\section{Corresponding author:}

Hiroaki Satoh, MD, Division of Respiratory Medicine, Mito Medical Center, University of Tsukuba, Mito, Ibaraki, 310-0015, Japan; e-mail: hirosato@md.tsukuba.ac.jp 